\title{
THE STATISTICAL MECHANICS OF THE SELF-GRAVITATING GAS: EQUATION OF STATE AND FRACTAL DIMENSION
}

\author{
H. J. de $\operatorname{Vega}^{(a)}$ and N. Sánchez ${ }^{(b)}$ \\ (a) LPTHE, Université Pierre et Marie Curie (Paris VI) et Denis Diderot (Paris VII), \\ Tour 16, 1er. étage, 4, Place Jussieu, 75252 Paris, Cedex 05, \\ FRANCE. Laboratoire Associé au CNRS UMR 7589. \\ (b) Observatoire de Paris, Demirm, 61, Avenue de l'Observatoire, \\ 75014 Paris, FRANCE. Laboratoire Associé au CNRS UA 336, \\ Observatoire de Paris et École Normale Supérieure.
}

(Dated: June 2000)

\begin{abstract}
We provide a complete picture of the self-gravitating non-relativistic gas at thermal equilibrium using Monte Carlo simulations (MC), analytic mean field methods (MF) and low density expansions. The system is shown to possess an infinite volume limit, both in the canonical (CE) and in the microcanonical ensemble (MCE) when $N, V \rightarrow \infty$, keeping $N / V^{1 / 3}$ fixed. We compute the equation of state (we do not assume it as is customary), the entropy, the free energy, the chemical potential, the specific heats, the compressibilities, the speed of sound and analyze their properties, signs and singularities. The MF equation of state obeys a first order non-linear differential equation of Abel type. The MF gives an accurate picture in agreement with the MC simulations both in the CE and MCE. The inhomogeneous particle distribution in the ground state suggest a fractal distribution with Haussdorf dimension $D$ with $D$ slowly decreasing with increasing density, $1 \lesssim D<3$.
\end{abstract}

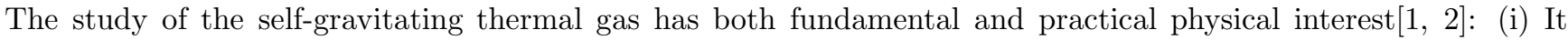
possess remarkable thermodynamic properties due to the long range of the gravitational force [such properties never occur in ordinary systems with short range forces]. (ii) It plays a central rôle in astrophysics and cosmology; cold clouds in the interstellar medium and its remarkable observed scaling laws (from $10^{-4} \mathrm{pc}$ up to $100 \mathrm{pc}$ ), as well as galaxy distributions (up to $100-200 \mathrm{Mpc}$ ) can be described with it. (iii) It is relevant for the study of stellar objects in the non-relativistic and relativistic cases.

The ground state is inhomogeneous and the usual thermodynamic limit: number of particles $N \rightarrow \infty$, volume $V \rightarrow \infty$, with $N / V$ fixed leads to collapse into a very dense phase. The gaseous phase can only exist when $N, V \rightarrow \infty$ with $N / V^{1 / 3}$ fixed. This is a diluted limit where the particle density $N / V$ goes to zero as $V^{-2 / 3}$. The appropriate dimensionless variable (for particles of mass $m$ at temperature $T$ on a box of volume $V$ ) is $\eta \equiv \frac{G m^{2} N}{L T}$ (it can be a spherical or cubic box with volume $V=L^{3}$ or $V=(4 \pi / 3) R^{3}$, respectively). $\eta$ is related to the Jeans' length of the gas $d_{J}$ through $\eta=3\left(L / d_{J}\right)^{2}$. We call 'thermodynamic limit' the limit $N \rightarrow \infty, R \rightarrow \infty$ with a fixed ratio $R / N$. In this limit, physical magnitudes are expressed naturally as functions of $\eta$.

For small $\eta$, the gas behaves as a perfect gas. For growing $\eta, P V / N T$ (where $P$ is the pressure) decreases (see figs.1-2) due to the attractive character of gravity. Finally, at some critical $\eta_{\text {crit }}$ the gas exhibits a sharp clumping transition to a dense phase with negative pressure. The extension of the gaseous phase and the value $\eta_{\text {crit }}$ depend on the thermodynamical ensemble (see figs.1-2): the gas phase is larger in the microcanonical ensemble (MCE) and smaller in the canonical ensemble (CE). We investigate the self-gravitating gas with Monte Carlo (MC) and analytic mean field (MF) methods; in the dilute limit we expand in powers of $\eta$. Our results show that the CE and MCE yield the same results in their common range of the gaseous phase. The MF correctly describes the thermodynamic limit except near the critical points, the MF is valid for $N\left|\eta-\eta_{\text {crit }}\right| \gg 1$. The vicinity of the critical point should be studied in a double scaling limit $N \rightarrow \infty, \eta \rightarrow \eta_{\text {crit }}$.

The particle distribution $\rho(\vec{q})$ proves to be inhomogeneous (except for $\eta \ll 1$ ) and described by an universal function of the geometry, $\eta$ and the ratio $\vec{r}=\vec{q} / R$. $D$ slowly decreases from the value $D=3$ for the ideal gas $(\eta=0)$ till $D=0.98$ in the extreme limit of the MC point taking the value 1.6 at $\eta_{\text {crit }}$ [see Table 1 ]. The particle density in the bulk behaves as $\rho(\vec{q}) \simeq r^{D-3}$. This indicates the presence of a fractal distribution with Haussdorf dimension $D$.

In the MCE, the entropy can be written as

$$
e^{S(E, N)_{M C E}}=\frac{1}{N !} \int \ldots \int \prod_{l=1}^{N} \frac{d^{3} p_{l} d^{3} q_{l}}{(2 \pi)^{3}} \delta\left[E-\sum_{l=1}^{N} \frac{p_{l}^{2}}{2 m}-U\left(\vec{q}_{1}, \ldots \vec{q}_{N}\right)\right]
$$

where $E$ is the total energy, $G$ is Newton's gravitational constant and

$$
U\left(\vec{q}_{1}, \ldots \vec{q}_{N}\right) \equiv-G m^{2} \sum_{1 \leq l<j \leq N} \frac{1}{\left|\vec{q}_{l}-\vec{q}_{j}\right|}
$$


In the CE the partition function can be written as

$$
\mathcal{Z}_{C E}=\frac{1}{N !} \int \ldots \int \prod_{l=1}^{N} \frac{d^{3} p_{l} d^{3} q_{l}}{(2 \pi)^{3}} e^{-\beta\left[\sum_{l=1}^{N} \frac{p_{l}^{2}}{2 m}+U\left(\vec{q}_{1}, \ldots \vec{q}_{n}\right)\right]} .
$$

We make now explicit the volume dependence by introducing the variables $\vec{r}_{l}, 1 \leq l \leq N$ as $\vec{q}_{l}=R \vec{r}_{l}, \vec{r}_{l}=$ $\left(x_{l}, y_{l}, z_{l}\right)$, where $\left|\vec{r}_{l}\right| \leq 1$ for a spherical box and $0 \leq x_{l}, y_{l}, z_{l} \leq 1$ for a cubic box. The momentum integrals can be straightforwardly computed both in eq.(11) and in eq.(2) yielding

$$
\begin{aligned}
& e^{S(E, N)_{M C E}}=\frac{(m N)^{3 N-2} R^{3 N / 2+1} G^{3 N / 2-1}}{N ! \Gamma\left(\frac{3 N}{2}\right) 2 \pi^{3 N / 2}} \int \ldots \int \prod_{l=1}^{N} d^{3} x_{l}\left[\xi+\frac{1}{N} u\left(\vec{r}_{1}, \ldots, \vec{r}_{N}\right)\right]_{+}^{3 N / 2-1} \\
& \mathcal{Z}_{C E}=\frac{1}{N !}\left(\frac{m T R^{2}}{2 \pi}\right)^{\frac{3 N}{2}} \int \ldots \int \prod_{l=1}^{N} d^{3} r_{l} e^{\eta u\left(\vec{r}_{1}, \ldots, \vec{r}_{N}\right)}
\end{aligned}
$$

where $u\left(\vec{r}_{1}, \ldots, \vec{r}_{N}\right) \equiv \frac{1}{N} \sum_{1 \leq l<j \leq N} \frac{1}{\left|\vec{r}_{l}-\vec{r}_{j}\right|}$ and $\xi \equiv \frac{E R}{G m^{2} N^{2}}$ are dimensionless and we introduced the notation $[X]_{+}^{n} \equiv$ $X^{n} \theta(X)$, being $\theta(X)$ the step function. All space integrals in eq.(3) and below are over an unit volume. The temperature is given in the MCE as a function of $E$ and $\xi$ by (for $N \gg 1$ )

$$
\begin{aligned}
\frac{1}{T}=\left(\frac{\partial S}{\partial E}\right)_{V}=\frac{N}{E} g(\xi), \frac{P V}{N T}=\frac{V}{N}\left(\frac{\partial S}{\partial V}\right)_{E}=\frac{1}{2}+\frac{1}{3} g(\xi) \\
g(\xi) \equiv \frac{\xi}{N} \frac{\partial}{\partial \xi} \log w(\xi, N), w(\xi, N) \equiv \int \ldots \int \prod_{l=1}^{N} d^{3} r_{l}\left[\xi+\frac{1}{N} u\left(\vec{r}_{1}, \ldots, \vec{r}_{N}\right)\right]_{+}^{3 N / 2-1}
\end{aligned}
$$

In the CE, the free energy results

$$
\begin{aligned}
& F=-T \log \mathcal{Z}_{C E}=F_{0}-T \Phi_{N}(\eta) \\
& \Phi_{N}(\eta)=\log \left[\int \ldots \int \prod_{l=1}^{N} d^{3} r_{l} e^{\eta u\left(\vec{r}_{1}, \ldots, \vec{r}_{N}\right)}\right]
\end{aligned}
$$

where $F_{0}=-N T \log \frac{e V}{N}\left(\frac{m T}{2 \pi}\right)^{3 / 2}$ is the free energy for an ideal gas. Defining $f(\eta) \equiv 1-\frac{\eta T}{3 V} \Phi_{N}^{\prime}(\eta)$, we then find

$$
\begin{aligned}
& P=-\left(\frac{\partial F}{\partial V}\right)_{T}=\frac{N T}{V} f(\eta), F=F_{0}-3 N T \int_{0}^{\eta} d x \frac{1-f(x)}{x} \\
& \frac{E}{N T}=-\frac{T}{N} \frac{\partial}{\partial T}\left(\frac{F}{T}\right)_{V}=3\left[f(\eta)-\frac{1}{2}\right] \\
& \frac{S}{N}=-\frac{3}{2}-\frac{F_{0}}{N T}+3 f(\eta)+3 \int_{0}^{\eta} d x \frac{1-f(x)}{x} .
\end{aligned}
$$

$P$ stands for the external pressure on the gas. Notice that the equation of state $P V=N T f(\eta)$ is here computed from the gravitational interaction, we do not assume an equation of state as it is customary. It follows from eqs. (4) and $(6)$ that $\eta \xi=g(\xi)=3[f(\eta)-1 / 2]$ and that the virial theorem, $\left(3 P V=\frac{3}{2} N T+E\right)$, holds both in the MCE and in the CE.

We find that the following properties hold in the $N \rightarrow \infty, L \rightarrow \infty$ limit provided $\eta$ and $\xi$ are kept fixed:

(a) $f(\eta)$ and $g(\xi)$ reach finite limits. [In fact, fixed $\eta$ implies a finite limit for $\xi$ and viceversa].

(b) The energy, free energy, entropy and $P V$ are proportional to $N$ (besides a $\log N$ term in the entropy as for an ideal gas).

(c) Both ensembles, the MCE and CE yield the same physical magnitudes.

We have verified properties (a)-(c) in three ways. First, by direct calculation in the dilute regime $(\xi \gg 1, \eta \ll 1)$ by expanding eqs.(4) and eqs.(5) in powers of $1 / \xi$ and $\eta$ for the MCE and CE, respectively. Second, by performing Monte Carlo simulations both in the MCE and the CE. Third, by mean field approximations to eqs.(4) and eqs.(5).

The specific heat at constant volume takes the form,

$$
c_{V}=\frac{T}{N}\left(\frac{\partial S}{\partial T}\right)_{V}=3\left[f(\eta)-\eta f^{\prime}(\eta)-\frac{1}{2}\right] .
$$


This expression is valid in the thermodynamic limit both in the CE and MCE. In the CE, the specific heat is related to the fluctuations of the potential energy $(\Delta U)^{2}$ and it is positive defined,

$$
\left(c_{V}\right)_{C E}=\frac{3}{2}+(\Delta U)^{2},(\Delta U)^{2} \equiv \frac{<U^{2}>-<U>^{2}}{N T^{2}}
$$

\section{Dilute Limit Calculations.}

For a dilute gas, we expand the integrands in eqs.(4) and eqs.(5) in powers of $1 / \xi$ or $\eta$, respectively. In the $N \rightarrow \infty$ limit the expressions simplify considerably. Moreover, divergent integrals in the zero cutoff limit are eliminated by $1 / N$ factors. That is, after the thermodynamic limit is taken the cutoff corrections are of the order $\mathcal{O}\left(a^{2}\right)$ and clearly vanish in the $a \rightarrow 0$ limit. We find after calculation,

$$
\begin{aligned}
& \frac{E}{N T}=g(\xi)=\frac{3}{2}-\frac{9 b_{0}}{2 \xi}-\frac{9}{4 \xi^{2}}\left(b_{1}-42 b_{0}^{2}\right)+\mathcal{O}\left(\xi^{-3}\right) \\
& \frac{P V}{N T}=f(\eta)=1-b_{0} \eta-\eta^{2}\left[\frac{1}{3} b_{1}-12 b_{0}^{2}\right]+\mathcal{O}\left(\eta^{3}\right) .
\end{aligned}
$$

where

$$
b_{0}=\frac{1}{6} \iint \frac{d^{3} r_{1} d^{3} r_{2}}{\left|\vec{r}_{1}-\vec{r}_{2}\right|}, b_{1}=\iint \frac{d^{3} r_{1} d^{3} r_{2} d^{3} r_{3}}{\left|\vec{r}_{1}-\vec{r}_{2}\right|\left|\vec{r}_{1}-\vec{r}_{3}\right|}
$$

For a sphere of unit volume we find $b_{0}^{s p h}=1 / 5, b_{1}^{s p h}=153 / 35$, whereas for a unit volume cube $b_{0}^{\text {cube }}=0.19462 \ldots$ Notice that the cube and sphere values differ only by about $3 \%$. We see from eq. (8) that the perfect gas behaviour gets corrected by negative definite terms due to the attractive nature of the gravitational interaction. [The full correction to the ideal gas, $\Phi_{N}^{\prime}(\eta)$, is positive definite according to eq.(5)].

\section{Monte Carlo Calculations.}

We applied the standard Metropolis algorithm in a cube of size $L$ with total energy $E$ in the MCE and at temperature $T$ in the CE. The number of particles $N$ went up to 2000[7]. We introduced a small short distance cutoff $\sim 10^{-3} L-10^{-6} L$ in the attractive Newton's potential. All results in the gaseous phase were insensitive to the cutoff value.

Two different phases show up: a non-perfect gas for $\eta<\eta_{c}$, and a condensed system with negative pressure for $\eta>\eta_{c}$. The transition between the two phases is very sharp, with a negative jump in the entropy from the gas to the condensed phase. This phase transition is associated with the Jeans instability.

We plot in figs.1-2 $f(\eta)=P V /[N T]$ as a function of $\eta$. For small $\eta$, the MC results for $P V /[N T]$ reproduce very well the analytical formula (8). $P V /[N T]$ monotonically decreases with $\eta$ as forecasted by the dilute expansion (8). For $\eta=\eta_{T} \simeq 1.51$ (point $T$ in fig.2), close to $\eta_{C} \simeq 1.54$ (point $C$ in fig.2) a phase transition suddenly happens in the $\mathrm{CE}$ and $P V /[N T]$ becomes large and negative. The interparticle distance $\langle r>$ monotonically decreases with $\eta$ too. When $\eta$ crosses $\eta_{T},<r>$ has a sharp decrease.

The MCE and CE Monte Carlo results are very close (up to the statistical error) for $0<\eta<\eta_{C}$, that is for $\infty>\xi>\xi_{C} \simeq-0.19$. In the MCE the gas does not clump at $\eta=\eta_{C}$ (point $C$ in fig.2) and the specific heat becomes negative between the points $C$ and $M C$. The gas does clump in the MCE at $\xi \simeq-0.32, \eta \simeq 1.35$ (point $M C$ in fig.2) increasing both its temperature and pressure discontinuously. As is clear, the domain between $C$ and $M C$ cannot be reached in the $\mathrm{CE}$ since $c_{V}>0$ in the $\mathrm{CE}$.

\section{Mean Field Calculations.}

We now recast the coordinate partition function $e^{\Phi_{N}(\eta)}$ as a functional integral in the thermodynamic limit.

$$
\begin{aligned}
& e^{\Phi_{N}(\eta)} \stackrel{N \geqq 1}{=} \iint D \rho d a e^{-N s[\rho(.)]+i a\left(\int d^{3} x \rho(\vec{x})-1\right)} \\
& s[\rho(.)]=-\frac{\eta}{2} \int \frac{d^{3} x d^{3} y}{|\vec{x}-\vec{y}|} \rho(\vec{x}) \rho(\vec{y})+\int d^{3} x \rho(\vec{x}) \log [\rho(\vec{x}) / e] .
\end{aligned}
$$

where we used the coordinates $\vec{x}$ in the unit volume. The first term is the potential energy, the second term is the functional integration measure for this case (see [3]). Eq.(9) is dominated for large $N$ by its stationary point solutions:

$$
\log \rho(\vec{x})-\eta \int \frac{d^{3} y \rho(\vec{y})}{|\vec{x}-\vec{y}|}=a,
$$


$a$ is a Lagrange multiplier enforcing the constraint $\int d^{3} x \rho(\vec{x})=1$. Applying the Laplacian and setting $\phi(\vec{x}) \equiv \log \rho(\vec{x})$ yields, for the spherically symmetric case

$$
\frac{d^{2} \phi}{d r^{2}}+\frac{2}{r} \frac{d \phi}{d r}+4 \pi \eta^{R} e^{\phi(r)}=0
$$

with boundary conditions $\phi^{\prime}(0)=0$ and $\phi^{\prime}(1)=-\eta^{R}$. Here the variable $\eta^{R}$ appropriate for a spherical symmetry is defined as $\eta^{R} \equiv \frac{G m^{2} N}{R T}=\eta\left(\frac{4 \pi}{3}\right)^{1 / 3}=1.61199 \ldots \eta$.

Using the scale covariance of eq.(11) [2], $\phi(r)$ can be expressed as $\phi(r)=\log \left(\lambda^{2} / 4 \pi \eta^{R}\right)+\chi(\lambda r)$ where

$$
\chi^{\prime \prime}(\lambda)+\frac{2}{\lambda} \chi^{\prime}(\lambda)+e^{\chi(\lambda)}=0, \chi^{\prime}(0)=0
$$

This equation is invariant under the transformation:

$$
\lambda \Rightarrow \lambda e^{\alpha} \quad \chi(\lambda) \Rightarrow \chi(\lambda)-2 \alpha,
$$

where $\alpha$ is a real number. Hence, we can set $\chi(0) \equiv 0$ without loosing generality.

$\chi(x)$ is independent of $\eta^{R}$ and $\lambda$ is related to $\eta^{R}$ by $\lambda \chi^{\prime}(\lambda)=-\eta^{R}$.

Evaluating the functional integral in eq.(9) by saddle point yields

$$
e^{\Phi_{N}\left(\eta^{R}\right)} \stackrel{N \gg 1}{=} \frac{e^{-N s\left(\eta^{R}\right)}}{\sqrt{D_{C}\left(\eta^{R}\right)}}\left[1+\frac{C\left(\eta^{R}\right)}{N}+\mathcal{O}\left(\frac{1}{N^{2}}\right)\right]
$$

where $D_{C}\left(\eta^{R}\right)$ stands for the determinant of small fluctuations around the spherically symmetric saddle point (11) and $C\left(\eta^{R}\right)$ for the two-loop corrections. $D_{C}\left(\eta^{R}\right)$ can be expressed as an infinite product over the partial waves $D_{C}\left(\eta^{R}\right)=\prod_{l \geq 0}\left[D_{l}\left(\eta^{R}\right)\right]^{2 l+1} \cdot D_{0}\left(\eta^{R}\right)$ and $D_{1}\left(\eta^{R}\right)$ can be computed in closed form [4]

$$
D_{0}\left(\eta^{R}\right)=\frac{1}{2 \eta^{R}}\left[\lambda^{2}\left(\eta^{R}\right) e^{\chi\left(\lambda\left(\eta^{R}\right)\right)}-\eta^{R}\right], D_{1}\left(\eta^{R}\right)=e^{\chi\left(\lambda\left(\eta^{R}\right)\right)} .
$$

$D_{0}\left(\eta^{R}\right)$ [see fig.1] is positive for $0 \leq \eta^{R} \leq \eta_{C}^{R}=2.51755 \ldots$ and vanish linearly in $\sqrt{\eta_{C}^{R}-\eta^{R}}$ at $\eta^{R}=\eta_{C}^{R}$.

We thus get for the free energy in the CE from eqs.(5), (9), (10) and (14)

$$
\begin{aligned}
& F=F_{0}+N T s\left(\eta^{R}\right)+\frac{T}{2} \log D_{C}\left(\eta^{R}\right)+\mathcal{O}\left(N^{-1}\right) \\
& \frac{P V}{N T}=f_{M F}\left(\eta^{R}\right)+\frac{\eta^{R} D_{C}^{\prime}\left(\eta^{R}\right)}{6 N D_{C}\left(\eta^{R}\right)}+\mathcal{O}\left(N^{-1}\right) \\
& f_{M F}\left(\eta^{R}\right) \equiv \frac{\lambda^{2}\left(\eta^{R}\right)}{3 \eta^{R}} e^{\chi\left(\lambda\left(\eta^{R}\right)\right)} \\
& s\left(\eta^{R}\right)=3\left[1-f_{M F}\left(\eta^{R}\right)\right]-\eta^{R}+\log \left[3 f_{M F}\left(\eta^{R}\right) / 4 \pi\right]
\end{aligned}
$$

It follows from eq. (12) and (16) that $f_{M F}\left(\eta^{R}\right)$ obeys the first order equation

$$
\eta^{R}(3 f-1) f^{\prime}\left(\eta^{R}\right)+\left(3 f-3+\eta^{R}\right) f=0 .
$$

which reduces to an Abel equation of first kind [6].

By expanding the solution of eq.(12) in powers of $\lambda$ one checks that eq.(15) reproduces eq.(8) (4]. We plot in fig.1 $f_{M F}\left(\eta^{R}\right)$ as a function of $\eta^{R}$ obtained by solving eqs. (12-15) by the Runge-Kutta method. We find that MC results (both in the MCE and $\mathrm{CE}$ ) and the MF results are in excellent agreement. (This happens although the geometry for the MC calculation is cubic while it is spherical for the MF).

The clumping phase transition takes place when $D_{C}\left(\eta^{R}\right)$ vanishes at $\eta^{R}=\eta_{C}^{R}$. At such point the expansion in $1 / N$ breaks down since the correction terms become large in eqs. 14 15. MF applies when $N\left|\eta_{C}^{R}-\eta^{R}\right| \gg 1$. Since,

$$
\frac{\eta^{R} D_{C}^{\prime}\left(\eta^{R}\right)}{6 D_{C}\left(\eta^{R}\right)} \stackrel{\eta^{R} \uparrow \eta_{C}^{R}}{=}-\frac{\eta_{C}^{R}}{3\left(\eta_{C}^{R}-\eta^{R}\right)} \rightarrow-\infty
$$

eq. 115) correctly suggests that $P V /[N T]$ becomes large and negative for $\eta^{R} \uparrow \eta_{C}^{R}$ in agreement with the MC results. 


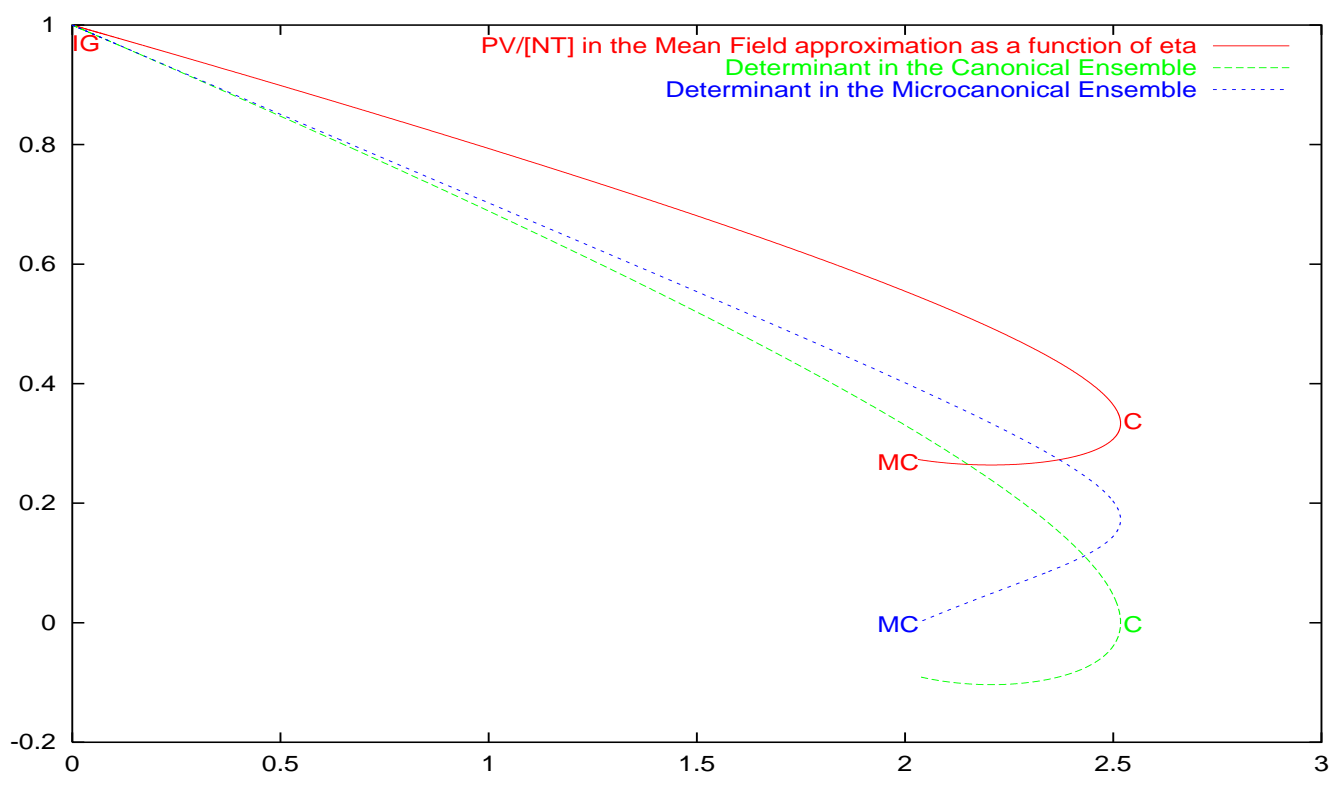

FIG. 1: $f_{M F}\left(\eta^{R}\right)=P V /[N T]$ as a function of $\eta^{R}$ in the MF approximation [eq.(15)], $D_{0}\left(\eta^{R}\right)$ for the canonical ensemble (CE) and $D_{M C}^{S}$ for the microcanonical ensemble (MCE).

From eqs.11-15) we obtain the following behaviour near the point $C$ in fig.1

$$
\begin{aligned}
& f_{M F}\left(\eta^{R}\right) \stackrel{\eta^{R} \uparrow \eta_{C}^{R}}{=} 1 / 3+0.213738 \ldots \sqrt{\eta_{C}^{R}-\eta^{R}}+\mathcal{O}\left(\eta_{C}^{R}-\eta^{R}\right) \\
&\left(c_{V}\right)_{M F} \stackrel{\eta^{R} \uparrow \eta_{C}^{R}}{=} 0.80714 \ldots\left(\eta_{C}^{R}-\eta^{R}\right)^{-1 / 2}-0.19924 \ldots+\mathcal{O}\left(\sqrt{\eta_{C}^{R}-\eta^{R}}\right)
\end{aligned}
$$

As noticed before, the $\mathrm{CE}$ only describes the region between the points $I G$ (ideal gas point, $\eta=0$ ) and $C$ in fig.1. The MCE goes beyond the point $C$ (till the point $M C$ ) with the physical magnitudes described by the second sheet of the square root in eqs.(17). We have between $C$ and $M C$

$$
\begin{aligned}
& f_{M F}\left(\eta^{R}\right) \stackrel{\eta^{R} \stackrel{\eta_{C}^{R}}{=}}{=} 1 / 3-0.213738 \ldots \sqrt{\eta_{C}^{R}-\eta^{R}}+\mathcal{O}\left(\eta_{C}^{R}-\eta^{R}\right) \\
& \left(c_{V}\right)_{M F} \stackrel{\eta^{R} \uparrow \eta_{C}^{R}}{=}-0.80714 \ldots\left(\eta_{C}^{R}-\eta^{R}\right)^{-1 / 2}-0.19924 \ldots+\mathcal{O}\left(\sqrt{\eta_{C}^{R}-\eta^{R}}\right)
\end{aligned}
$$

$c_{V}$ is negative in all the interval from $C$ to $M C$ and vanishes at the point $M C$.

A functional integral representation for $w(\xi, N)$ in the MCE follows by inverse Laplace transform of $e^{\Phi_{N}\left(\eta^{R}\right)}$ [ [ . The saddle point in the MCE is $N s\left(\eta^{R}\right)$ as in in the CE but the corrections are different. The S-wave determinant results, $D_{M C}^{S}=6 f_{M F}\left(\eta^{R}\right)^{2}-\left(11 / 2-\eta^{R}\right) f_{M F}\left(\eta^{R}\right)+1 / 2$. It is positive from $I G$ to $M C$ where it vanishes (fig.1).

\section{Speed of sound and compressibility.}

The isothermal compressibility and the speed of sound for long wavelengths 5 follow from the equation of state (6)

$$
\begin{gathered}
K_{T}=-\frac{1}{V}\left(\frac{\partial V}{\partial P}\right)_{T}=\frac{V}{N T} \frac{1}{f\left(\eta^{R}\right)+\frac{1}{3} \eta^{R} f^{\prime}\left(\eta^{R}\right)}, \\
\frac{v_{s}^{2}}{T}\left(\eta^{R}\right)=\frac{\left[f\left(\eta^{R}\right)-\eta^{R} f^{\prime}\left(\eta^{R}\right)\right]^{2}}{3\left[f\left(\eta^{R}\right)-\eta^{R} f^{\prime}\left(\eta^{R}\right)-\frac{1}{2}\right]}+f\left(\eta^{R}\right)+\frac{1}{3} \eta^{R} f^{\prime}\left(\eta^{R}\right) .
\end{gathered}
$$

$K_{T}$ is positive for $0 \leq \eta^{R}<\eta_{0}^{R}=2.4345 \ldots$ where $K_{T}$ diverges, it is negative for $\eta_{0}^{R}<\eta^{R}<\eta_{C}^{R}$. The singularity of $K_{T}$ before but near the point $C$ appears as a preliminary signal of the phase transition and perhaps $\eta_{0}^{R}$ is the transition point $T$ seen with the Monte Carlo simulations (see fig. 2). $K_{T}$ becomes positive between $C$ and $M C$.

The speed of sound squared $v_{s}^{2} / T\left(\eta^{R}\right)$, is positive and decreasing in the whole interval between $I$ and $C$. At the point $C$ it takes the value $v_{s}^{2} / T\left(\eta_{C}^{R}\right)=11 / 18$. Then, $v_{s}^{2} / T\left(\eta^{R}\right)$ decreases between $C$ and $M C$ becoming negative at $\eta_{1}^{R}=2.14674 \ldots v_{s}^{2}<0$ indicates an instability announcing the $M C$ critical point at $\eta_{m c}^{R}=2.03085 \ldots$ 


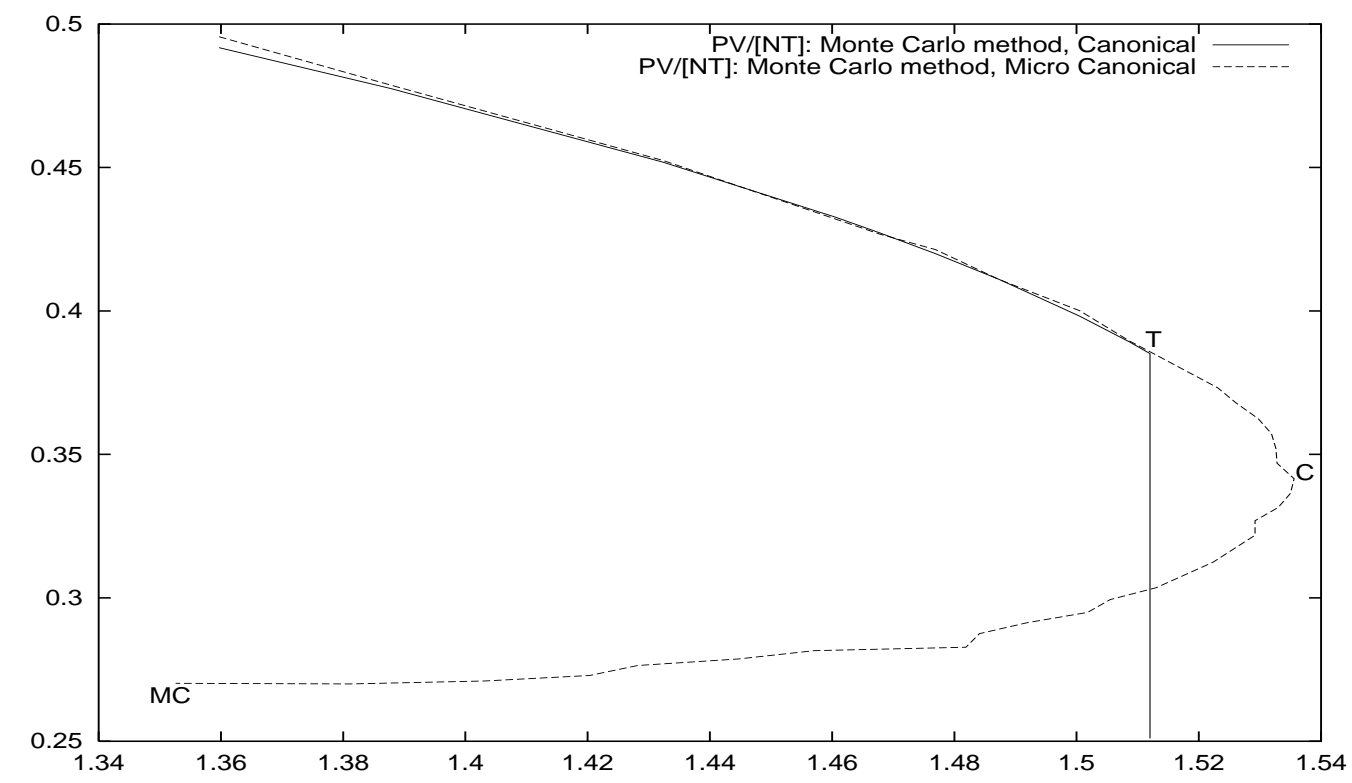

FIG. 2: $f(\eta)=P V /[N T]$ as a function of $\eta$ by Monte Carlo for the MCE and CE $(N=2000)$.

\section{Particle Distribution.}

In the dilute regime $\eta^{R} \ll 1$ the gas density is uniform, as expected. We find that these mass distributions approximately follow the power law

$$
\mathcal{M}(r) \simeq C r^{D}
$$

where $D$ slowly decreases with $\lambda\left(\eta^{R}\right)$ as depicted in Table 2 from the value $D=3$ for the ideal gas $\left(\eta^{R}=0\right)$ till $D=0.98$ in the extreme limit of the MC point.

The gravitational potential at the point $\vec{r}$ in the MF follows from eq.(10) to be

$$
U(\vec{r})=-\frac{T}{m}[\phi(\vec{x})-a] .
$$

The local pressure in the gas takes then the form $p(\vec{r})=T \rho(\vec{r})$. We have thus derived the equation of state for the self-gravitating gas. It turns to be locally the ideal gas equation. But, the self-gravitating gas is inhomogeneous, the pressure at the surface of a given volume is not equal to the temperature times the average density of particles in the volume. In particular, for the whole volume: $P V /[N T]=f\left(\eta^{R}\right) \neq 1$ (except for $\eta=0$ ).

\begin{tabular}{|l|c|l|}
\hline$\eta^{R}$ & $D$ & $C$ \\
\hline 0.1 & 2.97 & 1.0 \\
\hline$\eta_{G C}^{R}$ & 2.75 & 1.03 \\
\hline 2.0 & 2.22 & 1.1 \\
\hline$\eta_{C}^{R}$ & 1.60 & 1.07 \\
\hline$\eta_{M C}^{R}$ & 0.98 & 1.11 \\
\hline
\end{tabular}

TABLE 1. The Fractal Dimension $D$ and the proportionality coefficient $C$ as a function of $\eta^{R}$ from a fit to the mean field results according to $\mathcal{M}(r) \simeq C r^{D}$.

We have thus shown that the thermodynamics of the self-gravitating gas in the $N \rightarrow \infty, L \rightarrow \infty$ limit with $N / L$ fixed can be expressed in terms of a single function $f(\eta)$. Besides computing $f(\eta)$ numerically we obtained its Riemann sheet structure and its behaviour near the critical point analytically [eq.(17)].

[1] V. A. Antonov, Vest. Leningrad Univ. 7, 135 (1962). W. C. Saslaw, 'Gravitational Physics of Stellar and Galactic Systems', Cambridge U. Press, 1987. G. Horwitz and J. Katz, Ap. J. 222, 941 (1978). D. Lynden-Bell, cond-mat/9812172 and refs. 
therein. T. Padmanabhan, Phys. Rep. 188, 285 (1990). S. Chandrasekhar, An Introduction to the Study of the Stellar Structure, Univ. Chicago Press, 1939.

[2] H. J. de Vega, N. Sánchez and F. Combes, Nature, 383, 56 (1996), Phys. Rev. D54, 6008 (1996), Ap. J. 500, 8 (1998); in 'Current Topics in Astrofundamental Physics'. N. Sánchez and A. Zichichi editors, vol 511, Kluwer, 1998.

[3] L. N. Lipatov, JETP 45, 216 (1978)

[4] H. J. de Vega and N. Sánchez, in preparation.

[5] L. Landau and E. Lifchitz, Mécanique des Fluides, Eds. MIR, Moscou 1971.

[6] E. Kamke, Differentialgleichungen, Chelsea, NY, 1971.

[7] See for example, K. Binder and D. W. Heermann, Monte Carlo simulations in Stat. Phys., Springer series in Solid State, 80, 1988. 